\title{
Funciones Que Cumple El Cuaderno Resolutor Al Abordar Una Situación Problema ${ }^{1}$
}

\author{
Notebook That Meets Function Resolver \\ Problem By Addressing A Situation \\ Notebook que cumpre função Resolver \\ problema tratando A Situação
}

Recibido: mayo 2013

Aceptado: agosto 2013 sindy viviana rodriguez muriel ${ }^{2}$ yenifer yulieth rodriguez mayorga ${ }^{3}$

claudia patricia peña ramirez ${ }^{4}$ maryuri mosquera sanchez $z^{5}$

\section{Resumen}

Este artículo está especialmente elaborado para docentes y/o alumnos para profesores que deseen trabajar en la búsqueda de la solución de problemas a partir de la metodología del cuaderno Resolutor, partiendo de la experiencia en el aula con un grupo de estudiantes donde se lleva a cabo una situación - problema (lugares geométricos), haciendo énfasis en la resolución de problemas que se plantea desde la teoría y contrastando con la experiencia propia que se tiene para llegar a la solución del problema.se formulen preguntas: ¿Qué es un cuaderno resolutor?, ¿Cómo lo manejan los estudiantes?, ¿Qué instrucciones se le daban para escribir en el cuaderno?, clarifique el objetivo, defina y ejecuta el plan de acción y finalmente se evalúe la solución.

Palabras clave: Cuaderno resolutor; coordenadas polares; otras nociones de educación matemática; resolución de problemas; matemáticas escolares; geometría; cardioide; caracol de pascal.

\begin{abstract}
This article is specially designed for teachers and / or students for teachers who wish to work in the search for the solution of problems from Resolver Notebook methodology, based on experience in the classroom with a group of students which takes place a situation - problem (loci), with emphasis on solving problems that arise from the theory and in contrast to the experience that you have to get to the solution problema.se ask questions: What is a notebook resolver? , how do you handle the students, What gave instructions to write in the notebook?, clarify the objective, define and execute the action plan and finally evaluating the solution.
\end{abstract}

1 Artículo de Investigación

2 Universidad Distrital Francisco José de Caldas. Bogotá, Colombia. Contacto: sindy_viviana_2203@hotmail.com

3 Universidad Distrital Francisco José de Caldas. Bogotá, Colombia. Contacto: jenyuroma@yahoo.es

4 Universidad Distrital Francisco José de Caldas. Bogotá, Colombia. Contacto: claudia-_14@hotmail.com

5 Universidad Distrital Francisco José de Caldas. Bogotá, Colombia. Contacto: maryu1101@hotmail.com 
Keywords: Notebook resolver, polar coordinates, other notions of mathematics education, problem solving, school mathematics, geometry, cardioid, snail pascal.

\section{Resumo}

Este artigo é projetado especialmente para professores e / ou alunos para os professores que desejam trabalhar na busca de solução dos problemas de metodologia Notebook Resolver, com base na experiência em sala de aula com um grupo de estudantes que se realiza uma situação - problema (loci), com ênfase na resolução de problemas que surgem a partir da teoria e em contraste com a experiência que você tem que chegar ao problema. se solução perguntas: o que é um resolvedor de notebook? , como você lida com os estudantes, que deram instruções para escrever no caderno?, esclarecer o objetivo, definir e executar o plano de ação e, finalmente, avaliar a solução.

Palavras-chave: resolvedor Notebook, coordenadas polares, outras noções de educação matemática, resolução de problemas, a matemática da escola, geometria, cardióide, caracol pascal.

\section{Contextualización}

Para la solución de la situación planteada en el curso:

"Construir una circunferencia cualquiera, tomar un punto al azar sobre la circunferencia y por este trazar una recta tangente a la circunferencia, luego tomar un punto cualquiera y por este trazar una recta perpendicular a la recta tangente, punto de intersección $\mathrm{C}$ de las rectas. ¿Qué lugar geométrico hace $\mathrm{C}$ cuando se mueve el punto inicial A?"

Se ha tenido en cuenta llevar la investigación basada en el cuaderno resolutor, con el fin de identificar "las funciones que cumple el cuaderno resolutor al abordar una situación problema”.

\section{Referentes teóricos - prácticos}

La investigación se basó en el intento por definir la función que cumple el cuaderno resolutor en un proceso de resolución de problemas, para ello, se tiene en cuenta la teoría que permita clasificar como mínimo dicho objeto. Por tanto, se quiso fomentar en una situación particular; el desarrollo de los lugares geométricos, iniciando con la aclaración teórica de la temática.

Lugares geométricos desde la teoría: Un lugar geométrico es un conjunto de puntos que satisfacen una determinada condición. La solución de un problema de lugares geométricos es una ecuación (Becerra, s.f). Esto se toma dado que en la solución del problema es importante llegar a una ecuación que satisface la situación planteada (construcción).

En el abordaje que se ha tenido frente a la situación se han encontrado tres soluciones, de acuerdo a las condiciones que se puedan extraer del problema.

Circunferencia: La circunferencia es el lugar geométrico de todos los puntos de un plano que participan de la propiedad de equidistar de un punto fijo llamado centro (Villena Moisés, s.f).

Cardioide: La cardioide es la curva que dibuja un punto fijo $\mathrm{P}$ en un círculo que rueda sobre el exterior de un círculo fijo de igual tamaño, $\mathrm{O}$ bien, es el lugar geométrico de un punto fijo $\mathrm{P}$ en un círculo de radio $2^{\text {a }}$ que rueda en el interior de un círculo fijo de radio a, esta descripción recibe el nombre de generación doble (Fernández María, s.f). 
Caracol de Pascal: Es la concoide de la circunferencia. Es el lugar geométrico de los puntos M para los cuales $\mathrm{OM}=\mathrm{OP} \pm 1$

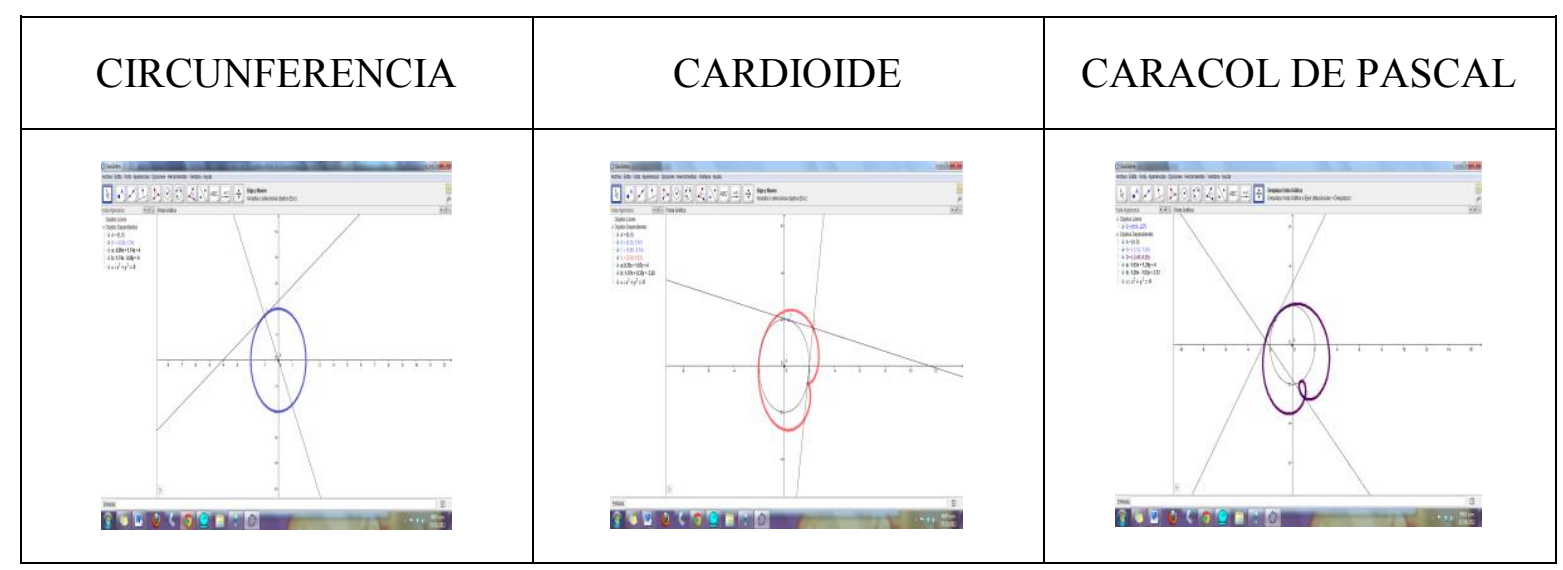

Fuente: elaboración propia

Lo que se ha mostrado anteriormente es la consulta realizada con respecto al problema cuando se abarcó en las dos primeras sesiones, pues dado a que la construcción se realizó en el programa de geogebra, esto permitió mostrar que lugar geométrico estaba dando la construcción.

Ahora bien ¿Cómo se refleja el cuaderno resolutor, la resolución de problemas en la situación? Unos de los documentos fuertes encontrados lo trata el docente Romero, J. (2011) en una publicación que hace acerca de: el cuaderno del resolutor mediador en la constitución de comunidades de práctica en la formación de profesores de matemáticas, ponencia que se presenta en Brasil; este artículo es un resultado de un proyecto de investigación ${ }^{6}$ financiado por la Universidad Distrital Francisco José de Caldas, en el año 2009. La investigación se enmarca en la preocupación por indagar cómo ocurre el aprendizaje de los estudiantes para profesor, cuando participan en entornos de aprendizaje diseñados desde la perspectiva sociocultural con el propósito de que aprendan a realizar la práctica de enseñar matemáticas.

Si se toma en cuenta los resultados plasmados en la investigación que realizó Romero, en el que muestra cual fue el proceso que tuvo la utilización del cuaderno como una norma de la clase y en lo que afirma, es que los estudiantes fueron incorporándolo como un instrumento de objetivación, individual y grupal, en el cual reconocen que su incorporación a la clase fue muy importante ya que les ayudo a llevar un orden del proceso que se llevaba:

..." logran conformar una comunidad de práctica que usa el cuaderno del resolutor no sólo como un activador de su "monitor interno" (Mason, J., Burton, L. \& Stacey, K., 1989) sino que lo convierten en un artefacto de mediación y comunicación colectiva, lo que es un indicador de la constitución”...

Se encuentra también un documento en el que no se menciona precisamente un cuaderno resolutor, pero el artículo refiere a carpetas de trabajo ya sean colectivas, carpetas de evaluación, carpetas en las que se muestra el trabajo que va desarrollando cada estudiante adjuntándolo al trabajo que se tiene en una carpeta, para la cual accede cualquier estudiante, esto de cierto modo tendría relación con el cuaderno en el sentido que no es un cuaderno individual el que lleva el proceso sino una carpeta grupal, colectiva que abarca el proceso de todos los estudiantes.

En cuanto al cuaderno de bitácora lo que presenta es como va evolucionando un estudiante con el apoyo del maestro para la superación profesional, y el papel que cumple el cuaderno de bitácora es 
llevar el proceso o desarrollo contando, reflexionando y analizando las cosas que se deben ir reforzando.

En cuanto a la metodología, Polya (1945), menciona cuatro fases que se deben tener en cuenta para la resolución de problemas, planteando un par de preguntas que oriente al docente para que se lleve a cabo en el proceso del estudiante; en la práctica, las preguntas orientan al estudiante del cómo y de qué manera puede ir desarrollándose el problema para encontrar resultados, pues inicialmente lo que se realiza es llevar al estudiante al pensar de que manera puede llegar a una solución con el conocimiento que se ha adquirido hasta el momento, ya cuando el estudiante no logra particularizar la situación, interviene el docente llevándolo a que investigue la manera con la cual se llegaría a una solución, teniendo en cuenta que todo proceso que se realice debe ir registrado en el cuaderno resolutor.

Durante el proceso de solución por parte de los estudiantes para profesores de matemáticas se emplearon dos categorías de orden cognitivo propias de la resolución de problemas: la herramienta heurística (Puig, 1996); donde el problema se convierte en uno similar ya que se empieza a formular en palabras más sencillas para ir resolviéndolo y la metacognición (Santos, 2007) basada en el aprendizaje y los conocimientos que se adquieren en los procesos cognitivos que se desarrollaron al momento de solucionar el problema.

\section{Descripción general de la experiencia en el aula}

El presente artículo está trabajado bajo el paradigma de investigación crítico y se toman de este, dos fases para apoyar y contrastar la teoría con lo aplicado en clase. Es decir con el modelo crítico implementado en el presente artículo se quiere mostrar y dar a conocer que el cuaderno resolutor se construye socialmente por la participación colectiva y las acciones transformadoras en el marco de enseñanza aprendizaje en el espacio de formación seminario de problemas I.
Este paradigma crítico de investigación implementado en el presente artículo tiene como propósito fundamental producir cambios en la función que cumple el cuaderno resolutor a la hora de abordar un problema; de este, se emplean dos fases: las técnicas y los instrumentos de recolección.

En cuanto a las técnicas es importante decir que se tuvo en cuenta: la observación, intervención tanto individual como grupal y análisis de documentos y los instrumentos de recolección; el cuaderno resolutor (en él, todas las etiquetas y avances de los problemas y talleres) y como evidencia de ello, fotografías del mismo.

Por último, es importante decir que las dos fases se aplican al momento de estar desarrollando actividades que requieren la resolución de problemas y que éstas son discutidas en el aula de clases a partir de las correspondientes bitácoras del cuaderno resolutor.

En este curso se ha llevado a cabo la solución de la situación planteada inicialmente, teniendo en cuenta parte de la teoría y las conjeturas obtenidas al realizar el procedimiento del problema, estos abordajes se han ido plasmando en un cuaderno resolutor donde hasta el momento se ha llevado un orden especifico en cuanto a lo realizado y a la teoría que lo sustenta.

El proceso que logró llevarse a cabo en el cuaderno resolutor, permitió dar solución a la situación problema planteada inicialmente por parte del docente, la dificultad que se presentó, fue el tratar de llevar al día este cuaderno, pues no evidenció de manera oportuna todo el proceso llevado, dado que el estudiante toma la decisión, si es adecuado mostrar un procedimiento incorrecto o de poner en evidencia los primeros pasos erróneos que se realizaron.

\section{Dificultades}

El cuaderno resolutor llevaba un orden moderado por el docente para que cada estudiante lo llevara correctamente, las ventajas del cuaderno es que las bitácoras muestran el crecimiento que se puede 
dar con la interacción de profesor-estudiante o a si mismo estudiante-estudiante, ya que permite analizar y reflexionar acerca de las ideas que surgen en el grupo, de igual manera se encontraron dificultades para desarrollar de manera adecuada el cuaderno resolutor:

- Una primera dificultad fue el tratar de interpretar la información que se tenía, dado que al realizar los procedimientos y ecuaciones encontradas, no había resultados.

- Al momento de analizar el proceso que se lleva en el cuaderno resolutor, encontramos por medio de la experiencia que no siempre por medio de los estudiantes se muestra todas las dificultades que se tiene en cuenta

- No siempre se toma en cuenta el cuaderno resolutor para mostrar el proceso que abarca al responder y desarrollar la situación problema.

\section{Logros}

- En el momento de generalizar la información y realizar inferencias se tiene en cuenta que el cuaderno Resolutor es un medio en el cual los procesos de aprendizaje son significativos al momento de dar solución a un problema.

- En algunos casos aunque el cuaderno Resolutor sirva para dar solución a un problema se convierte en un medio donde solo se da respuesta al problema pero pierde su función quizá de las etiquetas y de los procesos que se llevan a cabo para la finalización del problema.

- Las etiquetas en un cuaderno Resolutor son de gran importancia, puesto que dan paso a reflexiones acerca de la solución del problema y ayudan a aclarar o modificar estrategias para dar solución al problema.

- finalmente el cuaderno Resolutor cumple sus funciones si realmente quien lleve a cabo este proceso en la metodología de resolución de problemas los cumpla.

\section{Reflexión final}

En el proceso que se llevó a cabo en la resolución de problemas es de vital importancia de acuerdo a nuestra experiencia, plasmar cada uno de los procedimientos y/o estrategias llevadas en un cuaderno resolutor, el cual permita dar evidencia de la familiarización existente con el problema, es decir; la identificación de la situación de partida y de llegada teniendo en cuenta lo que se debe lograr, también la búsqueda de estrategias encaminadas a la solución del problema y por último se obtiene que el cuaderno resolutor se usa en proceso como instrumento de apoyo en la solución de problemas.

\section{Referencias}

Socorro C., (s.f), ponencia, El Aprendizaje basado en Proyectos en la Educación Matemática del siglo XXI Cuaderno de bitácora, canarias España.

Romero J., (2011), ponencia, El cuaderno del resolutor mediador en la constitución de comunidades de práctica en la formación de profesores de matemáticas, Recife Brasil.

Teoría crítica y formación del profesorado, articulo de internet, recuperado el 14 de noviembre del 2012, en la web: http://educritica. idoneos.com/index.php/Teor\%C3\%ADa_ critica_de_la_educaci\%C3\%B3n_y_formaci\%C3\%B3n_del_profesorado\#13_Crear_ la_carpeta_con_el_\%E2\%80\%9CDiario $\%$ E2\%80\%9D_de_aula_Est\%C3\%A1_ escrito_por_el_alumnado_de_forma_ voluntaria\%2C_el_debate_sobre_los_criterios_de_evaluaci\%C3\%B3n_y_clasificaci\%C3\%B3n_del_alumnado_implicado_en_la_ clase\%E2\% 80\%A6_y_otros_aspectos_que_ los_colaboradores_puedan_proponer_y_ resultan_interesantes. 